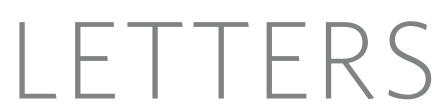

\title{
Enhancing CD8 T-cell memory by modulating fatty acid metabolism
}

\author{
Erika L. Pearce ${ }^{1}$, Matthew C. Walsh ${ }^{1}$, Pedro J. Cejas ${ }^{1}$, Gretchen M. Harms ${ }^{1}$, Hao Shen ${ }^{2}$, Li-San Wang ${ }^{1,3}$, \\ Russell G. Jones ${ }^{4}$ \& Yongwon $\mathrm{Choi}^{1}$
}

CD8 T cells, which have a crucial role in immunity to infection and cancer, are maintained in constant numbers, but on antigen stimulation undergo a developmental program characterized by distinct phases encompassing the expansion and then contraction of antigen-specific effector $\left(\mathrm{T}_{\mathrm{E}}\right)$ populations, followed by the persistence of long-lived memory $\left(T_{M}\right)$ cells $s^{1,2}$. Although this predictable pattern of CD8 T-cell responses is well established, the underlying cellular mechanisms regulating the transition to $T_{M}$ cells remain undefined ${ }^{1,2}$. Here we show that tumour necrosis factor (TNF) receptor-associated factor 6 (TRAF6), an adaptor protein in the TNF-receptor and interleukin-1R/Toll-like receptor superfamily, regulates $\mathrm{CD} 8 \mathrm{~T}_{\mathrm{M}}$-cell development after infection by modulating fatty acid metabolism. We show that mice with a T-cell-specific deletion of TRAF6 mount robust CD8 $\mathrm{T}_{\mathrm{E}}$-cell responses, but have a profound defect in their ability to generate $T_{M}$ cells that is characterized by the disappearance of antigen-specific cells in the weeks after primary immunization. Microarray analyses revealed that TRAF6-deficient CD8 T cells exhibit altered expression of genes that regulate fatty acid metabolism. Consistent with this, activated CD8 T cells lacking TRAF6 display defective AMP-activated kinase activation and mitochondrial fatty acid oxidation (FAO) in response to growth factor withdrawal. Administration of the anti-diabetic drug metformin restored
FAO and CD8 $\mathrm{T}_{\mathrm{M}}$-cell generation in the absence of TRAF6. This treatment also increased CD8 $\mathrm{T}_{M}$ cells in wild-type mice, and consequently was able to considerably improve the efficacy of an experimental anti-cancer vaccine.

A prevailing paradigm in immunology is that antigenic signal strength drives progressive T-cell differentiation ${ }^{3}$. To investigate this model regarding $\mathrm{T}_{\mathrm{M}}$ cells, we studied CD8 T-cell responses to bacterial infection in mice with a T-cell-specific deletion of TRAF6 (TRAF6- $\Delta \mathrm{T}$ ), a negative regulator of antigen-specific T-cell activation $^{4}$. Despite having fewer total CD8 T cells (Supplementary Fig. 1), TRAF6- $\Delta \mathrm{T}$ mice mounted normal ovalbumin (OVA)-specific $\mathrm{T}_{\mathrm{E}^{-}}$ cell responses to attenuated Listeria monocytogenes expressing OVA (LmOVA; Fig. 1a and Supplementary Fig. 2). To examine CD8 $\mathrm{T}_{\mathrm{M}}$ cells in TRAF6- $\Delta$ T mice, we immunized with LmOVA and measured OVA-specific cells 60 days post-infection. Although OVA-specific $\mathrm{T}_{\mathrm{E}}$-cell responses were intact, CD8 $\mathrm{T}_{\mathrm{M}}$-cell generation in TRAF6$\Delta \mathrm{T}$ mice was severely compromised (Fig. 1b and Supplementary Fig. 3), even with tenfold higher or lower immunizing doses (not shown). In mice lacking $c b l-b^{5}$, a different negative regulator of antigen-specific $\mathrm{T}$-cell activation, $\mathrm{T}_{\mathrm{M}}$ cells developed normally (Supplementary Fig. 4), indicating that failure of $\mathrm{T}_{\mathrm{M}}$-cell generation in TRAF6- $\Delta \mathrm{T}$ mice cannot be entirely explained by loss of a negative regulator.

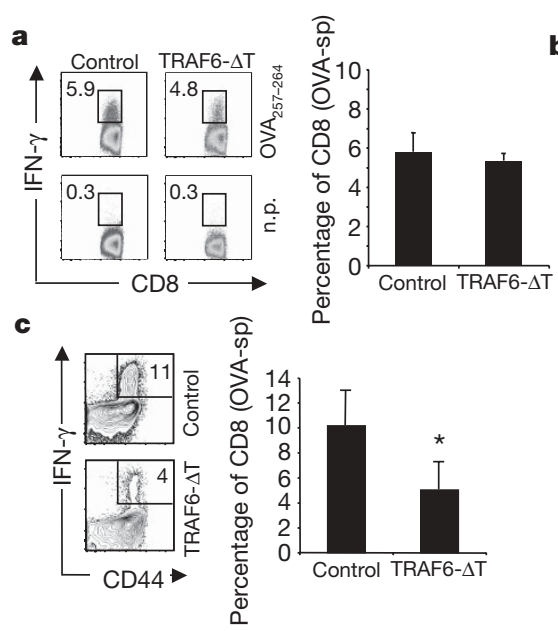

Figure 1 | TRAF6- $\Delta$ T mice mount normal $T_{E}$-cell responses, but have impaired $\mathbf{T}_{\mathbf{M}}$-cell development after immunization with $\boldsymbol{L}$. monocytogenes. Control and TRAF6- $\Delta$ T mice were LmOVA-immunized and spleen (a and c) or spleen (SPL), lymph nodes (LN), and bone marrow (BM) (b) cells were restimulated with OVA peptide and analysed for intracellular interferon- $\gamma$ (IFN- $\gamma$ ) 7 days (a, $n=3-5$ per group) or 60 days (b, $n=3$ per group)

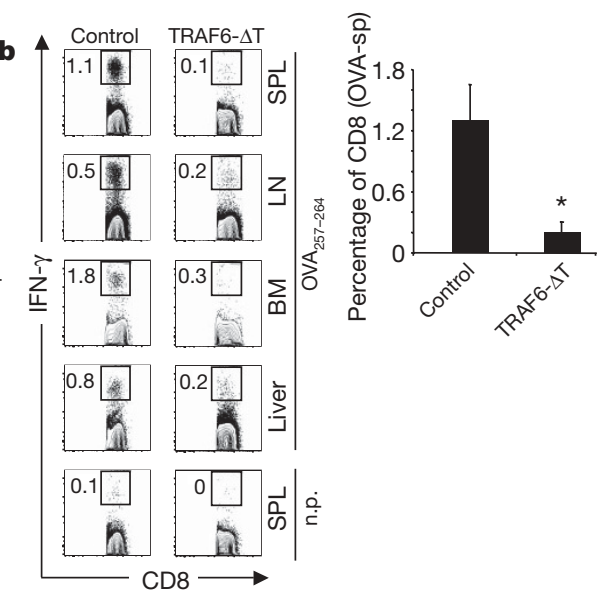

post-infection. n.p., no peptide. c, Sixty days post-immunization mice were challenged with LmOVA, and OVA-specific cells were analysed 7 days postchallenge ( $n=3$ per group). Dot plots and bar graphs show percentages of CD8 $\mathrm{T}$ cells producing IFN $-\gamma$ (means and standard deviation); $* \mathrm{P}=0.02$ (b), 0.0005 (c). 
A hallmark of $\mathrm{T}_{\mathrm{M}}$ cells is the ability to mount accelerated recall responses to challenge infection. To confirm the impaired generation of CD8 $\mathrm{T}_{\mathrm{M}}$ cells in TRAF6- $\Delta \mathrm{T}$ mice, we challenged previously immunized control and TRAF6- $\Delta \mathrm{T}$ mice with LmOVA and measured OVA-specific cells 7 days later. TRAF6- $\Delta \mathrm{T}$ mice failed to respond robustly to re-infection, indicating that $T_{M}$ cells may not have been generated and that the smaller OVA-specific population present in TRAF6- $\Delta$ T mice was a new primary response (Fig. 1c). To determine whether TRAF6- $\Delta \mathrm{T}$ mice generate $\mathrm{T}_{\mathrm{M}}$ cells, we transferred equal numbers of OVA-specific cells from previously immunized control and TRAF6- $\Delta \mathrm{T}$ mice 28 days post-infection, at which time a small population of OVA-specific cells appeared to be present (Supplementary Fig. 3), and compared functionality on a per cell basis in response to challenge. We reasoned that naive donor cells transferred into immune-competent animals would not respond to infection because they would be vigorously outcompeted by endogenous naive cells, and only $\mathrm{T}_{\mathrm{M}}$ donor cells would mount accelerated recall responses that could outcompete endogenous naive cells. At day 7 post-challenge, donor-derived OVA-specific secondary $\mathrm{T}_{\mathrm{E}}$ cells from control mice represented $11 \%$ of the engrafted population whereas TRAF6- $\Delta \mathrm{T}$ donor cells were undetectable (Supplementary Fig. 5), demonstrating a severe impairment in CD8 $\mathrm{T}_{\mathrm{M}^{-} \text {-cell develop- }}$ ment in TRAF6- $\Delta \mathrm{T}$ mice.

TRAF6- $\Delta$ T mice accumulate CD4 T cells and develop multi-organ inflammatory disease $\mathrm{e}^{4}$. Because CD4 T cells can influence CD8 T-cell responses $^{6-8}$ we wanted to rule out effects from the TRAF6-deficient CD4 T-cell compartment. To determine whether defective CD8 $\mathrm{T}_{\mathrm{M}^{-}}$ cell generation in TRAF6- $\Delta \mathrm{T}$ mice was intrinsic to CD8 $\mathrm{T}$ cells, we crossed TRAF6- $\Delta \mathrm{T}$ mice with major-histocompatibility-complexclass-I-restricted OVA-specific T-cell-receptor-transgenic OT-I mice. Because OT-I mice lack CD4 T cells, generation of OTITRAF6- $\Delta \mathrm{T}$ mice allowed us to assay a pure population of uniformly naive TRAF6-deficient CD8 T cells (Supplementary Figs 6-8). We transferred OTI-TRAF6-wild type (WT) and OTI-TRAF6- $\Delta$ T cells into congenic recipients, which possess a normal CD4 T-cell compartment, and then immunized with LmOVA (Fig. 2a). By transferring only small numbers of transgenic cells we could measure endogenous OVA-specific cells at the same time as the engrafted donor response, allowing us to assume that most donor cells were activated ${ }^{9}$. In addition, following $\mathrm{T}$-cell responses by serially bleeding allowed us to compare the kinetics of responding CD8 T cells within individual animals. Although both OTI-TRAF6-WT and OTITRAF6- $\Delta \mathrm{T}$ donor cells mounted strong $\mathrm{T}_{\mathrm{E}}$-cell responses (days 4-6), OTI-TRAF6- $\Delta$ T cells were not maintained during contraction (days 10-21) and were undetectable by 3 weeks post-infection (Fig. 2b, c and Supplementary Fig. 9). Donor $\mathrm{T}_{\mathrm{E}}$ cells from both genotypes exhibited similar expression of classical activation markers after primary immunization (Supplementary Figs 10 and 11); however, consistent with published data regarding killer cell lectin-like receptor 1 (KLRG1) as a marker for short-lived $\mathrm{T}_{\mathrm{E}}$ cells that do not form $\mathrm{T}_{\mathrm{M}}$ cells, a greater percentage of the OTI-TRAF6- $\Delta \mathrm{T} \mathrm{T}_{\mathrm{E}}$-cell population expressed higher levels of KLRG1 (Supplementary Fig. $12)^{10}$. After challenge infection, only donor OTI-TRAF6-WT T $\mathrm{M}_{\mathrm{M}}$ cells responded robustly (Fig. 2b, c and Supplementary Fig. 9). These results demonstrate that, although TRAF6 is dispensable for CD8 $\mathrm{T}_{\mathrm{E}}$-cell responses, TRAF6 signalling within CD8 $\mathrm{T}$ cells is crucial to $\mathrm{T}_{\mathrm{M}}$-cell generation.

Antigen-specific CD8 $\mathrm{T}$ cells responding to infection are not a homogenous population, but consist of small numbers of $\mathrm{T}_{\mathrm{M}^{-} \text {-cell }}$ precursors that express interleukin 7 receptor (IL7R $\alpha$; ref. 11). In conjunction with this idea, numerous studies have delineated roles for the $\gamma_{c}$ cytokines IL7 and IL15 as critical factors for CD8 T-cell homeostasis and $\mathrm{T}_{\mathrm{M}}$-cell development ${ }^{12-15}$. Impaired $\gamma_{c}$ cytokine signalling in TRAF6-deficient CD8 T cells could explain the defect in their ability to form $\mathrm{T}_{\mathrm{M}}$ cells. We tested the ability of CD8 $\mathrm{T}_{\mathrm{E}}$ cells to respond to IL15 and found that both OTI-TRAF6-WT and OTITRAF6- $\Delta \mathrm{T}$ cells exhibit similar responsiveness to this cytokine a
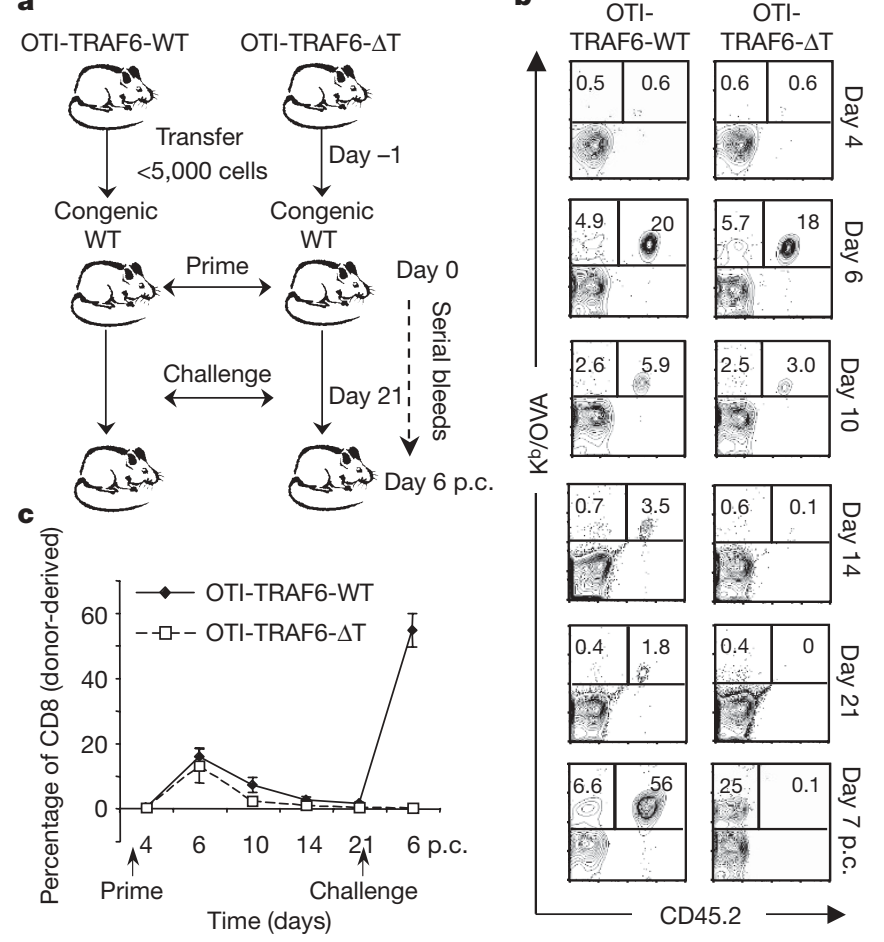

e

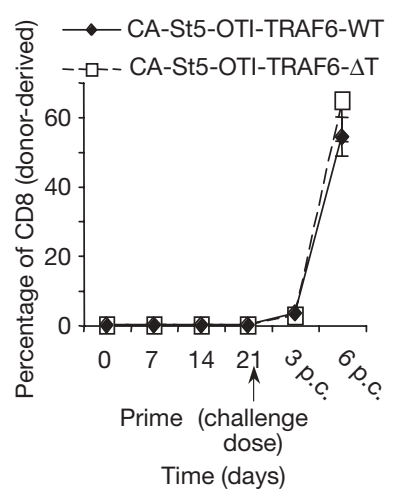

Figure 2 | TRAF6 intrinsically regulates $C D 8 \mathbf{T}_{\mathbf{M}}$-cell generation. OT-I cells $(<5,000)$ from OTI-TRAF6-WT and OTI-TRAF6- $\Delta \mathrm{T}(\mathrm{CD} 45.2 ; \mathbf{a}-\mathbf{c})$ or CASt5-OTI-TRAF6-WT and CA-St5-OTI-TRAF6- $\Delta$ T mice (CD45.2; d, e) were transferred into CD45.1 recipients ( $n=5-9$ per group) and LmOVAimmunized. Three weeks post-transfer, immune (a-d) or unimmunized (e) mice were LmOVA-challenged. Mice were bled as indicated and cells were surface-stained. Dot plots show donor cells by CD45.2 and $\mathrm{K}^{\mathrm{b}} / \mathrm{OVA}$ tetramer (numbers indicate total CD8 T-cell percentages that are host- or donorderived). Line graphs represent percentages of donor-derived CD8 T cells (means \pm standard deviation). p.c., post challenge.

(Supplementary Figs 13 and 14). To be sure that the lack of $\mathrm{T}_{\mathrm{M}}$-cell development by TRAF-6-deficient CD8 T cells is not secondary to a defect in survival, and to investigate further a possible defect in $\gamma_{c}$ cytokine signalling, we crossed OTI-TRAF6- $\Delta \mathrm{T}$ mice to mice constitutively expressing an active form of Stat-5 (also known as Stat5b; CASt5 $)^{16}$, a critical signalling molecule downstream of the $\gamma_{c}$ cytokine receptor ${ }^{16}$. CD8 T cells expressing CA-St5 show augmented survival, proliferation and B-cell leukemia/lymphoma $2(\mathrm{Bcl} 2)$ expression ${ }^{17}$, and CA-St5 mice exhibit selective expansion of memory-like CD8 T cells ${ }^{16}$. We transferred CA-St5-OTI-TRAF6-WT and CA-St5-OTITRAF6- $\Delta \mathrm{T}$ cells into congenic mice, immunized, and followed the donor and host OVA-specific responses (Fig. 2d and Supplementary Fig. 15). Contraction of CA-St5-OTI-TRAF6-WT populations was reduced compared to control OT-I populations (Fig. 2c, d). Surprisingly, however, CA-St5-OTI-TRAF6- $\Delta$ T cells were not rescued from contracting to undetectable levels 3 weeks post-infection (Fig. $2 \mathrm{~d}$ 
and Supplementary Fig. 15). Importantly, we confirmed that CA-St5 expression in TRAF6-deficient CD8 T cells enhanced survival in an ex vivo survival assay (Supplementary Fig. 16), indicating that the severe contraction exhibited by the TRAF6-deficient OT-I population (Fig. 2c, d and Supplementary Fig. 15) and the ensuing lack of $\mathrm{T}_{\mathrm{M}^{-}}$ cell development is not secondary to a general survival defect. Animals primed with the challenge dose of bacteria 3 weeks post-transfer showed no differences in the $\mathrm{T}_{\mathrm{E}}$-cell response between the genotypes (Fig. 2e and Supplementary Fig. 15), indicating that the loss of TRAF6-deficient CD8 T cells is exaggerated in response to signals after infection, and not simply due to defects in homeostatic signals after transfer.

We used a systems biology approach to identify unique gene expression signatures between OTI-TRAF6-WT and OTI-TRAF6$\Delta \mathrm{T}$ CD8 $\mathrm{T}$ cells that could account for their differences in survival after infection. We performed microarray analyses for both genotypes 6 and 10 days post-transfer and infection (Supplementary Fig. 17). Owing to similar survival rates between OTI-TRAF6-WT and OTI-TRAF6- $\Delta \mathrm{T}$ CD8 $\mathrm{T}$ cells during the peak of the $\mathrm{T}_{\mathrm{E}}$-cell response, but striking differences in survival during contraction, we reasoned that expression of genes related to survival might begin to diverge between genotypes at day 10 post-infection. We used the NIAID DAVID website (http://david.abcc.ncifcrf.gov) to look for pathways in the KEGG database that have significant overlaps with differentially expressed genes in our microarray experiment. We discovered that TRAF6-deficient cells from day 10 post-infection displayed defects in the expression of genes that function in several metabolic pathways, including fatty acid metabolism (Fig. 3a).

FAO is an important survival pathway in metabolically stressed cells $^{18}$ and the removal of signals associated with infection, such as growth factor cytokines including IL2, induces metabolic stress in haematopoietic cells ${ }^{19,20}$. Therefore, we tested the ability of OTITRAF6-WT and OTI-TRAF6- $\Delta \mathrm{T}$ CD8 $\mathrm{T}$ cells to oxidize fatty acids

\begin{tabular}{|c|c|c|c|c|}
\hline Group & $\begin{array}{l}\text { No. of genes } \\
\text { up or down }\end{array}$ & $\begin{array}{l}\text { No. of } \\
\text { KEGG } \\
\text { genes }\end{array}$ & $\begin{array}{l}\text { Percentage } \\
\text { of input }\end{array}$ & $\begin{array}{c}\text { No. of } \\
\text { pathways }\end{array}$ \\
\hline Day 6 up & 39 & 8 & 20 & 0 \\
\hline Day 6 down & 52 & 12 & 23 & 0 \\
\hline Day 10 up & 38 & 6 & 15 & 0 \\
\hline \multirow[t]{2}{*}{ Day 10 down } & 54 & 12 & 22 & (3) \\
\hline & & $\longrightarrow$ & & \\
\hline \multicolumn{2}{|c|}{ KEGG pathway } & $\begin{array}{l}\text { No. of } \\
\text { genes }\end{array}$ & $\begin{array}{c}\text { Percentage } \\
\text { of input }\end{array}$ & $P$ value \\
\hline \multicolumn{2}{|c|}{$\begin{array}{l}\text { Valine, leucine and } \\
\text { isoleucine degradation }\end{array}$} & 3 & 5.6 & 0.0044 \\
\hline \multicolumn{2}{|c|}{ Fatty acid metabolism } & 3 & 5.6 & 0.0046 \\
\hline \multicolumn{2}{|c|}{ Bile acid biosynthesis } & 2 & 3.7 & 0.078 \\
\hline
\end{tabular}
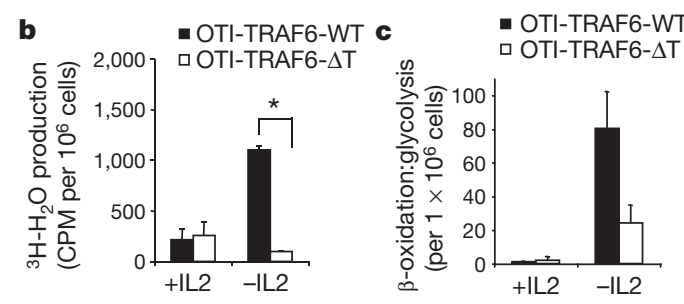

Figure 3 | TRAF6-deficient CD8 T cells display defects in fatty acid metabolism that can be corrected by metformin. a, OT-I cells $(<5,000)$ from OTI-TRAF6-WT and OTI-TRAF6- $\Delta$ T mice (CD45.2) were transferred into CD45.1 recipients and immunized with LmOVA. Six ( $n=3$ per group) and 10 ( $n=5$ per group) days post-infection, donor cells were analysed by microarray (a and Supplementary Fig. 17). Tables were generated using NIAID DAVID and KEGG databases. b, FAO (measured as mitochondrial $\beta$-oxidation) of activated OTI-TRAF6-WT and OTI-TRAF6- $\Delta$ T cells postIL2 withdrawal; ${ }^{*} P=0.012$. c, Ratio of $\beta$-oxidation to glycolysis in activated after withdrawal of the pro-proliferative growth factor cytokine IL2 in vitro. After IL2 withdrawal, only control OTI-TRAF6-WT CD8 T cells increased FAO to a high degree (Fig. 3b). Consistent with normal activation (Supplementary Fig. 18), activated control and TRAF6deficient CD8 T cells increased glycolysis in response to IL2 (data not shown). Also, TRAF6-deficient CD8 T cells displayed normal upregulation of FAO in response to glucose withdrawal, indicating that cells are not completely unfit in the absence of TRAF6, but rather their inability to regulate catabolic fatty acid metabolism is due to the lack of a specific signal from growth factor (Supplementary Fig. 19). Importantly, although transgenic expression of CA-St 5 did enhance T-cell survival after infection (Supplementary Fig. 16), it did not rescue the defect in FAO in TRAF6-deficient cells after growth factor withdrawal (Supplementary Fig. 20), indicating that FAO defects are not secondary to survival defects.

Proliferating T cells use glycolytic metabolism rather than FAO for energy generation ${ }^{21-23}$, and growth factors enable this bias ${ }^{24}$. In the absence of glucose, cells can survive in part by ceasing proliferation and switching to catabolic metabolism like FAO and autophagy ${ }^{18,20}$. OTI-TRAF6- $\Delta \mathrm{T}$ CD8 $\mathrm{T}$ cells were notably less capable of effecting this metabolic switch than OTI-TRAF6-WT CD8 T cells after IL2 withdrawal (Fig. 3c). This indicated that TRAF6-deficient CD8 T cells responding to infection are unable to survive contraction and persist as long-lived $\mathrm{T}_{\mathrm{M}}$ cells due to an inability to properly engage pathways of FAO when growth factors such as IL2 become limiting after the peak of the immune response.

AMP-activated kinase (AMPK) and Akt are central regulators of FAO and glycolyis, respectively ${ }^{23}$. Consistent with reduced FAO, TRAF6-deficient CD8 T cells have lower active AMPK levels after IL2 withdrawal (Fig. 3d). To investigate whether AMPK defects could underlie decreased FAO in TRAF6-deficient CD8 T cells, we measured the effect of the anti-diabetic drug metformin, which promotes AMPK activation $^{25}$, on FAO after IL2 withdrawal. Metformin increased

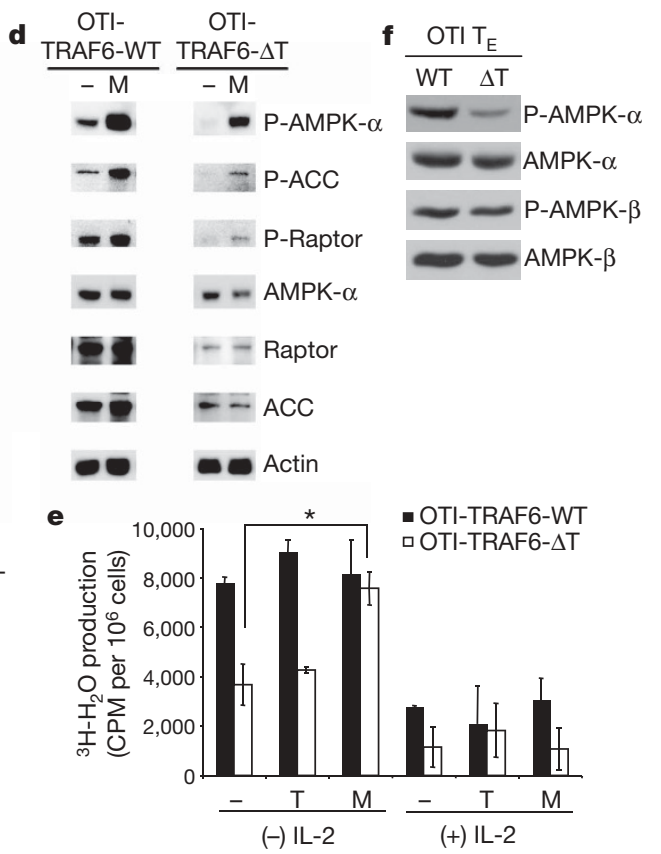

OTI-TRAF6-WT and OTI-TRAF6- $\Delta$ T cells post-IL2 withdrawal. $\mathbf{d}$, Western analysis of cells $16 \mathrm{~h}$ post-IL2 withdrawal with and without metformin (M; acetyl CoA carboxylase (ACC, also known as Acaca) and raptor are examined as targets of AMPK). e, Mitochondrial $\beta$-oxidation of activated OTI-TRAF6WT and OTI-TRAF6- $\Delta$ T cells with and without IL2, where cells received nothing $(-)$, triciribine $(\mathrm{T})$ or metformin $(\mathrm{M})$ for $16 \mathrm{~h},{ }^{*} P=0.003$.

f, Western analysis of purified OTI-TRAF6-WT and OTI-TRAF6- $\Delta \mathrm{T} \mathrm{T}_{\mathrm{E}}$ donor cells (7 days post-infection). All error bars show mean and standard deviation. 
AMPK activation (Fig. 3d) and rescued FAO in TRAF6-deficient CD8 T cells (Fig. 3e). In contrast, FAO in TRAF6-deficient cells was unaffected by the Akt inhibitor triciribine (Fig. 3e), indicating that Akt may not regulate this metabolic pathway. These data support that FAO initiation after the peak of infection, accompanied by the reduction in $\mathrm{T}$-cell survival signals, underlies $\mathrm{T}_{\mathrm{M}}$-cell development, and that commitment to this metabolic pathway is affected when TRAF6 is absent. In such a case, stimulating FAO in TRAF6-deficient CD8 $\mathrm{T}$ cells would rescue the observed defects and promote $\mathrm{T}_{\mathrm{M}}$ cells. Consistent with this hypothesis, OTI-TRAF6- $\Delta \mathrm{T}$ CD8 $\mathrm{T}$ cells had lower levels of active AMPK at the peak of the $\mathrm{T}_{\mathrm{E}}$-cell response (Fig. $3 \mathrm{f}$ ).

To explore whether inducing FAO in vivo would rescue the observed defects in TRAF6-deficient CD8 T cells and promote $\mathrm{T}_{\mathrm{M}}$ cells, we administered metformin daily after T-cell transfer and infection. Remarkably, metformin promoted survival not only of OTI-TRAF6- $\Delta \mathrm{T}$ CD8 $\mathrm{T}$ cells throughout contraction but also of endogenous OVA-specific cells, resulting in enhanced $\mathrm{T}_{M}$ cells with the capacity to respond to re-infection (Fig. 4a and Supplementary Fig. 21). Metformin also promoted OTI-TRAF6-WT CD8 T-cell survival (Fig. 4b). Treatment with a second FAO-inducing drug ${ }^{26-28}$, the mTOR (mammalian target of rapamycin, also known as FRAP1) inhibitor rapamycin, resulted in markedly increased $T_{M}$-cell development and ensuing recall response for both OTI-TRAF6- $\Delta$ T and OTI-TRAF6-WT CD8 T cells (Fig. 4a, b and Supplementary Fig. 21). Together these data strongly point towards the commitment to FAO as a key requirement for $\mathrm{T}_{\mathrm{M}}$-cell development, and the pharmacological modulation of this metabolic pathway as a potential target for vaccine design.

a

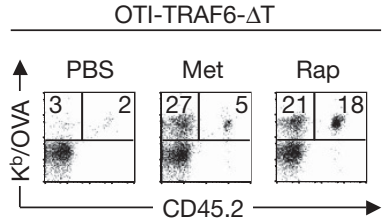

b OTI-TRAF6-WT
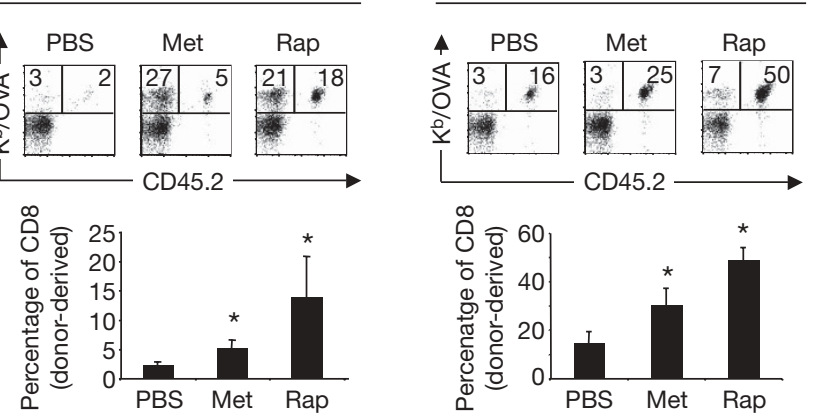

c

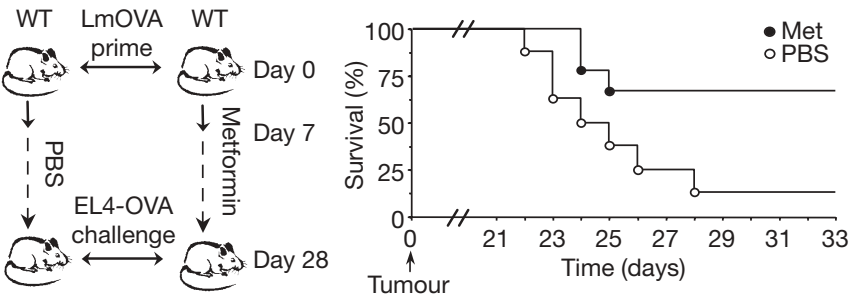

Figure 4 | Metformin treatment promotes $\mathbf{T}_{M}$-cell generation and protective immunity after infection and tumour challenge. a, b, OT-I cells $(<5,000)$ from OTI-TRAF6-WT and OTI-TRAF6- $\Delta$ T mice $(C D 45.2)$ were transferred into CD45.1 recipients and immunized with LmOVA. Eight days post-infection, mice were injected daily with $\mathrm{PBS}$ ( $n=7-9$ per group), metformin ( $n=7-9$ per group) or rapamycin ( $n=5$ per group) for 3 weeks and were then challenged with LmOVA. OVA-specific responses of host and donor cells in the blood were measured 5 days post-challenge. Dot plot numbers reflect the percentages of total CD8 T cells that are host- (left) or donor- (right) derived (OVA-specific). Bar graphs represent the percentage of CD8 T cells that are donor-derived (means and standard error). ${ }^{*} P$ values (comparing to PBS) are 0.015 (Met) and 0.000038 (Rap) (a), and 0.0373 (Met) and 0.00028 (Rap) (b). c, C57BL/6 mice were immunized and daily injections of metformin or PBS began 7 days post-infection. Three weeks later, treatments ceased and mice were inoculated with EL4-OVA tumours. Tumours became palpable by 18 days post-inoculation and mice were killed when tumours reached $2 \mathrm{~cm}$. The graph reflects percentage survival $(n=9$, metformin; $n=8$, PBS).
Promoting CD8 $\mathrm{T}_{\mathrm{M}^{-}}$-cell development is a major goal of vaccination. Our data showing increased CD8 $\mathrm{T}_{\mathrm{M}}$ cells for donor OT-I and endogenous OVA-specific cells after metformin treatment indicated that commitment to FAO is generally important for CD8 $\mathrm{T}_{\mathrm{M} \text {-cell }}$ development, and not only in settings of TRAF6-deficiency. This led us to the idea that pharmacological modulation of T-cell metabolism could enhance vaccine efficacy. To test this we used an experimental vaccine against an aggressive tumour ${ }^{29}$. We immunized control mice with LmOVA and then began daily injections of PBS or metformin after the peak of the $\mathrm{T}_{\mathrm{E}}$-cell response. After 3 weeks, drug treatment ceased and mice were inoculated with EL4-OVA tumour cells (Fig. 4c). Metformin was administered after $\mathrm{T}_{\mathrm{E}}$-cell expansion to avoid the possibility of altering the $\mathrm{T}_{\mathrm{E}}$-cell response. To rule out direct effects of metformin on tumour growth, treatment was stopped $24 \mathrm{~h}$ before tumour injection. After EL4-OVA inoculation, 6 out of 9 metformin-treated mice survived $>33$ days compared to only 1 out of 8 PBS-treated mice (Fig. 4c). This increase in survival after metformin treatment correlated with an increase in $T_{M}$ cells before tumour inoculation (Supplementary Fig. 22), indicating that metformin treatment enhanced $\mathrm{T}_{\mathrm{M}}$-cell generation resulting in greater protective anti-tumour immunity.

Proliferating T cells use glucose as their main energy source and suppress fatty acid metabolism, whereas quiescent cells (that is, naive and $\mathrm{T}_{\mathrm{M}}$ ) break down fatty acids, amino acids and glucose interchangeably for energy ${ }^{23}$. As such, proliferating $\mathrm{T}_{\mathrm{E}}$ cells display an anabolic signature typified by increased glycolytic metabolism, whereas $T_{M}$ cells display a catabolic signature. This implies that during contraction there is an active conversion of cellular energy metabolism to generate $\mathrm{T}_{\mathrm{M}}$ cells. Current models suggest that during contraction T cells compete for growth factors such as IL2, and we speculate that as these growth factors become limiting cells become stressed and undergo a metabolic transformation essential for $\mathrm{T}_{\mathrm{M}}$-cell generation. We propose a model in which TRAF6 has a critical role after infection by regulating a metabolic switch in CD8 T cells that promotes survival and development into long-lived $\mathrm{T}_{\mathrm{M}}$ cells.

Investigating the role of TRAF6 in $\mathrm{T}_{\mathrm{M}}$-cell development led us to the surprising finding that energy metabolism can be pharmacologically manipulated during an immune response to promote CD8 $\mathrm{T}_{\mathrm{M}^{-}}$ cell generation and protective immunity. Although the exact mechanism of metformin-mediated CD8 $\mathrm{T}_{\mathrm{M}}$-cell enhancement remains unclear, our work indicates that metabolism-altering drugs hold promise as immunotherapeutics and command further study as modulators of T-cell responses. Metformin has been shown to inhibit tumour progression ${ }^{25,30}$, although given our results it is unclear how metformin-regulated changes in $\mathrm{T}_{\mathrm{M}}$ cells contribute to its antitumour properties. Our findings highlight a critical link between metabolic transitions and cell fate determination, and may have important implications for therapeutic and prophylactic vaccine development.

\section{METHODS SUMMARY}

TRAF $6{ }^{\text {flox/flox }}$ CD4-cre (-) (control) and TRAF6 ${ }^{\text {flox/llox }}$ CD4-cre(+) (TRAF6$\Delta \mathrm{T}$ ) were bred in-house (backcrossed more than ten generations to $\mathrm{C} 57 \mathrm{BL} / 6$ ) and have been previously described ${ }^{4}$. C57BL/6 (WT) and OT-I transgenic mice were purchased from The Jackson Laboratory. OT-I mice were bred to TRAF $6{ }^{\text {flox/flox }} \mathrm{CD} 4$-cre mice to generate control OT-I-TRAF $6{ }^{\text {lox/flox }} \mathrm{CD} 4$ cre $(-)$ or OT-I-TRAF6 ${ }^{\text {flox/++}}$ CD4-cre $(+)$ mice (OTI-TRAF6-WT) and OT-ITRAF $6{ }^{\text {flox/flox }} \mathrm{CD} 4-\mathrm{cre}(+)(\mathrm{OTI}-\mathrm{TRAF} 6-\Delta \mathrm{T})$. Mice expressing the constitutively active Stat 5 transgene $(\mathrm{CA}-\mathrm{St} 5)^{16}$ were provided by M. Farrar and bred to OT-ITRAF $6^{\text {flox } /+} \mathrm{CD} 4-\mathrm{cre}(+)$ mice to generate control OT-I-CA-St5TRAF $6^{\text {flox/flox }}$ CD4-cre $(-)$ mice (OTI-CA-St5-TRAF6-WT) and OT-I-CA-St5TRAF6 ${ }^{\text {flox/flox }} \mathrm{CD} 4-\mathrm{cre}(+)$ mice (OTI-CA-St5-TRAF6- $\left.\Delta \mathrm{T}\right)$. Age-matched control and TRAF6- $\Delta \mathrm{T}$ mice were injected intravenously (i.v.) with a sub-lethal dose of $1 \times 10^{6}$ colony forming units (CFU) of recombinant attenuated L. monocytogenes (LmOVA) for primary immunizations and challenged i.v. with $1 \times 10^{7}$ CFU for secondary immunizations. CD45.1 congenic mice from OT-I adoptive transfer experiments were injected with a sub-lethal dose of $5 \times 10^{6} \mathrm{CFU}$ of LmOVA for primary immunizations and were challenged i.v. with $5 \times 10^{7}$ 
CFU for secondary immunizations. Acute LmOVA infections are resolved and bacteria are cleared by day 7 in all mice. All $P$ values were calculated using unpaired two-tailed Student's $t$-test.

Full Methods and any associated references are available in the online version of the paper at www.nature.com/nature.

\section{Received 23 January; accepted 23 April 2009.}

Published online 3 June 2009.

1. Harty, J. T. \& Badovinac, V. P. Shaping and reshaping CD $8^{+}$T-cell memory. Nature Rev. Immunol. 8, 107-119 (2008)

2. Prlic, M., Williams, M. A. \& Bevan, M. J. Requirements for CD8 T-cell priming, memory generation and maintenance. Curr. Opin. Immunol. 19, 315-319 (2007).

3. Lanzavecchia, A. \& Sallusto, F. Progressive differentiation and selection of the fittest in the immune response. Nature Rev. Immunol. 2, 982-987 (2002).

4. King, C. G. et al. TRAF6 is a T cell-intrinsic negative regulator required for the maintenance of immune homeostasis. Nature Med. 12, 1088-1092 (2006).

5. Bachmaier, K. et al. Negative regulation of lymphocyte activation and autoimmunity by the molecular adaptor Cbl-b. Nature 403, 211-216 (2000).

6. Shedlock, D. J. \& Shen, H. Requirement for CD4 T cell help in generating functional CD8 T cell memory. Science 300, 337-339 (2003).

7. Sun, J. C. \& Bevan, M. J. Defective CD8 T cell memory following acute infection without CD4 T cell help. Science 300, 339-342 (2003).

8. Janssen, E. M. et al. $\mathrm{CD4} 4^{+} \mathrm{T}$ cells are required for secondary expansion and memory in $\mathrm{CD}^{+}{ }^{+}$lymphocytes. Nature $421,852-856$ (2003).

9. Badovinac, V. P., Haring, J. S. \& Harty, J. T. Initial T cell receptor transgenic cell precursor frequency dictates critical aspects of the $\mathrm{CD} 8^{+} \mathrm{T}$ cell response to infection. Immunity 26, 827-841 (2007).

10. Joshi, N. S. et al. Inflammation directs memory precursor and short-lived effector $\mathrm{CD}^{+} \mathrm{T}$ cell fates via the graded expression of $\mathrm{T}$-bet transcription factor. Immunity 27, 281-295 (2007).

11. Kaech, S. M. et al. Selective expression of the interleukin 7 receptor identifies effector CD8 T cells that give rise to long-lived memory cells. Nature Immunol. 4 , 1191-1198 (2003)

12. Schluns, K. S., Williams, K., Ma, A., Zheng, X. X. \& Lefrancois, L. Cutting edge: requirement for IL-15 in the generation of primary and memory antigen-specific CD8 T cells. J. Immunol. 168, 4827-4831 (2002)

13. Schluns, K. S., Kieper, W. C., Jameson, S. C. \& Lefrancois, L. Interleukin-7 mediates the homeostasis of naive and memory CD8 T cells in vivo. Nature Immunol. 1, 426-432 (2000)

14. Tan, J. T. et al. Interleukin (IL)-15 and IL-7 jointly regulate homeostatic proliferation of memory phenotype $\mathrm{CD} 8^{+}$cells but are not required for memory phenotype CD4 ${ }^{+}$cells. J. Exp. Med. 195, 1523-1532 (2002).

15. Surh, C. D., Boyman, O., Purton, J. F. \& Sprent, J. Homeostasis of memory T cells. Immunol. Rev. 211, 154-163 (2006)

16. Burchill, M. A. et al. Distinct effects of STAT5 activation on $\mathrm{CD} 4^{+}$and $\mathrm{CD} 8^{+} \mathrm{T}$ cell homeostasis: development of $\mathrm{CD} 4^{+} \mathrm{CD} 25^{+}$regulatory $\mathrm{T}$ cells versus $\mathrm{CD} 8^{+}$ memory T cells. J. Immunol. 171, 5853-5864 (2003).
17. Kelly, J. et al. A role for Stat 5 in $C D 8^{+}$T cell homeostasis. J. Immunol. 170, 210-217 (2003).

18. Buzzai, M. et al. The glucose dependence of Akt-transformed cells can be reversed by pharmacologic activation of fatty acid beta-oxidation. Oncogene 24 , 4165-4173 (2005).

19. Rathmell, J. C., Vander Heiden, M. G., Harris, M. H., Frauwirth, K. A. \& Thompson, C. B. In the absence of extrinsic signals, nutrient utilization by lymphocytes is insufficient to maintain either cell size or viability. Mol. Cell 6, 683-692 (2000).

20. Lum, J. J. et al. Growth factor regulation of autophagy and cell survival in the absence of apoptosis. Cell 120, 237-248 (2005).

21. Frauwirth, K. A. et al. The CD28 signaling pathway regulates glucose metabolism. Immunity 16, 769-777 (2002).

22. Jones, R. G. et al. The proapoptotic factors Bax and Bak regulate T cell proliferation through control of endoplasmic reticulum $\mathrm{Ca}^{2+}$ homeostasis. Immunity 27, 268-280 (2007).

23. Jones, R. G. \& Thompson, C. B. Revving the engine: signal transduction fuels $T$ cell activation. Immunity 27, 173-178 (2007).

24. Fox, C. J., Hammerman, P. S. \& Thompson, C. B. Fuel feeds function: energy metabolism and the T-cell response. Nature Rev. Immunol. 5, 844-852 (2005)

25. Buzzai, M. et al. Systemic treatment with the antidiabetic drug metformin selectively impairs p53-deficient tumor cell growth. Cancer Res. 67, 6745-6752 (2007).

26. Sipula, I. J., Brown, N. F. \& Perdomo, G. Rapamycin-mediated inhibition of mammalian target of rapamycin in skeletal muscle cells reduces glucose utilization and increases fatty acid oxidation. Metabolism 55, 1637-1644 (2006).

27. Peng, T., Golub, T. R. \& Sabatini, D. M. The immunosuppressant rapamycin mimics a starvation-like signal distinct from amino acid and glucose deprivation. Mol. Cell. Biol. 22, 5575-5584 (2002).

28. Brown, N. F., Stefanovic-Racic, M., Sipula, I. J. \& Perdomo, G. The mammalian target of rapamycin regulates lipid metabolism in primary cultures of rat hepatocytes. Metabolism 56, 1500-1507 (2007).

29. Moore, M. W., Carbone, F. R. \& Bevan, M. J. Introduction of soluble protein into the class I pathway of antigen processing and presentation. Cell 54, 777-785 (1988).

30. Huang, X. et al. Important role of the LKB1-AMPK pathway in suppressing tumorigenesis in PTEN-deficient mice. Biochem. J. 412, 211-221 (2008).

Supplementary Information is linked to the online version of the paper at www.nature.com/nature.

Acknowledgements We thank C. Krawczyk, C. King, M. Farrar, Y. Paterson, Z.-K. Pan and E. J. Pearce. This work is supported in part by grants from the NIH (Y.C., H.S.), CIHR (R.G.J.) and an NCl Institutional Training Grant (E.L.P.).

Author Contributions E.L.P., M.C.W., R.G.J. and Y.C. designed the research and analysed the data. E.L.P., M.C.W., P.J.C., L.W. and G.M.H. performed experiments. E.L.P., M.C.W., H.S., R.G.J. and Y.C. contributed to the preparation of the manuscript. E.L.P. wrote the manuscript with M.C.W. and Y.C.

Author Information Microarray data is in the public repository Gene Expression Omnibus (GEO) under accession number GSE15750. Reprints and permissions information is available at www.nature.com/reprints. Correspondence and requests for materials should be addressed to Y.C. (ychoi3@mail.med.upenn.edu). 


\section{METHODS}

Mice. $\mathrm{Cblb}^{-1-}$ mice were a gift from J. Penninger. B6.Ly5.2/Cr (CD45.1 congenic) mice were purchased from the National Cancer Institute. All animals were cared for according to the Animal Care Guidelines of the University of Pennsylvania.

Immunizations. We used the attenuated strain of recombinant LmOVA deleted for actA (LmOVA) ${ }^{31}$ throughout this paper for convention, however, challenging control and TRAF6- $\Delta \mathrm{T}$ mice with $1 \times 10^{6} \mathrm{CFU}$ of non-attentuated recombinant Listeria expressing $\mathrm{OVA}^{32}$, or priming and challenging CD45.1 congenic mice from an OT-I adoptive transfer with $1 \times 10^{5} \mathrm{CFU}$ and $1 \times 10^{6}$ of recombinant Listeria expressing OVA, respectively, yielded similar results. To determine bacterial clearance, control and TRAF6- $\Delta \mathrm{T}$ were immunized with $8 \times 10^{6} \mathrm{CFU}$ LmOVA and CFU per spleen and liver were determined 2, 4 and 6 days after infection as described ${ }^{31}$. A total of $5 \times 10^{6}$ EL4-OVA (EG.7) tumour cells were injected into the right flank of mice as indicated.

Flow cytometry and intracellular cytokine staining. All fluorochromeconjugated monoclonal antibodies were purchased from BD Pharmingen or eBioscience. All staining was performed as previously described ${ }^{31}$. For ex vivo intracellular cytokine staining, cells were cultured at $37^{\circ} \mathrm{C}$ for $5 \mathrm{~h}$ in complete medium supplemented with $100 \mathrm{U} \mathrm{ml}^{-1}$ of recombinant human IL2 and $1.0 \mu \mathrm{l} \mathrm{ml}^{-1}$ GolgiStop, in either the presence or the absence of $\mathrm{OVA}_{257-264}$ peptide at $1.0 \mu \mathrm{g} \mathrm{ml}^{-1}$. OVA-specific $\mathrm{CD}^{+} \mathrm{T}$ cells were also quantified by direct staining with $\mathrm{H} 2-\mathrm{K}^{\mathrm{b}} / \mathrm{OVA}_{257-264}\left(\mathrm{~K}^{\mathrm{b}} / \mathrm{OVA}\right) \mathrm{MHC}$-peptide tetramers, either by killing animals and collecting cells from organs or by collecting blood from a live animal (serial bleeds), as indicated.

Adoptive transfers. Splenocytes from control and TRAF6- $\Delta$ T mice 28 days postimmunization were stained with $\mathrm{K}^{\mathrm{b}} / \mathrm{OVA}$ tetramer to determine numbers of OVA-specific CD8 T cells. Splenocytes containing $1 \times 10^{4}$ of OVA-specific cells were transferred intravenously to recipient mice followed by challenge infection as indicated. For OT-I cell adoptive transfers, OT-I cells were obtained from the blood or the spleen and then stained with $\mathrm{K}^{\mathrm{b}} / \mathrm{OVA}$ tetramers to determine numbers of OT-I cells, and then $<5,000$ OT-I cells were transferred into recipient mice. Adoptive transfer experiments were done with either OTITRAF6 ${ }^{\text {flox/flox }} \mathrm{CD} 4-\mathrm{cre}(-)$ or OTI-TRAF6 ${ }^{\text {flox/+ }} \mathrm{CD} 4-\mathrm{cre}(+)$ cells, and this confirmed that CD4-cre-expressing cells are not rejected.

In vitro $\mathrm{T}$-cell stimulation. Naive $\mathrm{T}$ cells were activated with anti-CD3 $\left(1.0 \mu \mathrm{g} \mathrm{ml}^{-1}\right)$, anti-CD28 $\left(0.5 \mu \mathrm{g} \mathrm{ml}^{-1}\right)$ and $100 \mathrm{U} \mathrm{ml}^{-1}$ of IL2 for 3 or 4 days and then assayed as indicated.

Metabolism assays. All activated T cells were rested in the absence of IL2 for 3-4 h (rest period) before being cultured in the absence or presence of IL2 or glucose (withdrawal period). The glycolytic rate of $\mathrm{T}$ cells was determined by measuring the conversion of $5{ }^{3} \mathrm{H}$-glucose to tritiated water, as described previously $^{33}$. In brief, in vitro activated OT-I cells were plated at $1 \times 10^{6}$ per $\mathrm{ml}$ in 24 well plates and cultured with and without glucose and with and without IL2 $\left(100 \mathrm{U} \mathrm{ml}^{-1}\right)$ as indicated for 14-16 h. Cells were collected, washed twice and resuspended in either glucose-free or glucose-replete medium with and without $100 \mathrm{U} \mathrm{ml}^{-1}$ of IL2 (RPMI, Gibco), and then incubated with $10 \mu \mathrm{m} \mathrm{Ci}$ of $5-{ }^{3} \mathrm{H}-$ glucose (Perkin-Elmer) at $37^{\circ} \mathrm{C}$ for $1 \mathrm{~h}$. The reaction was stopped by adding $\mathrm{HCl}$ $\left(0.1 \mathrm{M}\right.$ final). ${ }^{3} \mathrm{H}_{2} \mathrm{O}$ generated by enolase activity was separated from $5-{ }^{3} \mathrm{H}$ glucose by diffusion, and counts were measured using a 1450 Microbeta scintillation counter (Wallac). Measurement of mitochondrial-dependent $\beta$-oxidation of fatty acids was conducted as previously described with modifications ${ }^{34}$. In brief, $1 \times 10^{6}$ activated OT-I cells were cultured with and without glucose and/or with and without IL2 $\left(100 \mathrm{U} \mathrm{ml}^{-1}\right)$ as indicated for $14-16 \mathrm{~h} .\left[9,10-{ }^{3} \mathrm{H}\right]$-palmitic acid complexed to BSA (essentially fatty acid free; Sigma) was added to the cultures and incubated for $6-8 \mathrm{~h}$. Mitochondrial-independent $\beta$-oxidation was assessed by measuring $\left[9,10-{ }^{3} \mathrm{H}\right]$-palmitate oxidation in the presence of $200 \mu \mathrm{M}$ etomoxir (Sigma), an irreversible inhibitor of carnitine palmitoyltransferase I (CPT1), the rate-limiting enzyme for mitochondrial import of long-chain fatty acids. Supernatant was applied to ion-exchange columns (Dowex 1X8-200, Sigma), and ${ }^{3} \mathrm{H}_{2} \mathrm{O}$ recovered by eluting the columns with water. One-fifth of the recovered ${ }^{3} \mathrm{H}_{2} \mathrm{O}$ was then used for scintillation counting. The rate of $\beta$ oxidation was calculated as the difference between oxidation counts in the presence or absence of etomoxir, and expressed as CPM per $1 \times 10^{6}$ cells.
Metformin $(2 \mathrm{mM})^{35}$ or triciribine $(1 \mu \mathrm{M})^{36}$ was added during the IL2 withdrawal period as indicated.

In vivo drug treatment. Mice were injected daily with $300 \mu \mathrm{l}$ of PBS or metfor$\min \left(250 \mathrm{mg} \mathrm{kg}^{-1}\right)^{35}$ in $300 \mu \mathrm{l}$ of PBS. Rapamycin was dissolved in PBS/5\% DMSO and injected daily $\left(1.5 \mathrm{mg} \mathrm{kg}^{-1}\right)^{37}$. The PBS vehicle for these experiments also contained 5\% DMSO. No effect between PBS and PBS/5\% DMSO was detected.

Microarray. After OT-I cell adoptive transfer and LmOVA infection, splenocytes and blood were collected (at day 6 and 10 post-infection) from individual mice and donor cells were sorted (FACS Vantage, BD Bioscience) on the CD45.2 donor marker directly into Trizol LS (Invitrogen). RNA was extracted and samples were analysed using the Affymetrix Mouse Genome 4302.0 Array at the University of Pennsylvania Microarray Core Facility. We first used the gcrma package ${ }^{38}$ from the Bioconductor software ${ }^{39}$ to generate $\log _{2}$ probesets expression levels. Hierarchical clustering showed that one of the samples is an outlier and is excluded in subsequent analyses. We used the limma software package ${ }^{40}$ from Bioconductor to assess the effect of time (days 6 and 10) and genotype (WT and Traf $6^{-1-}$ ) by fitting the expression levels of each probeset $i$ using R model specification 'expr $\sim$ genotype * timepoint', which translates to the following model:

$$
x_{i j}=m_{i}+a_{i} G_{i j}+b_{i} T_{i j}+r_{i} G T_{i j}+e_{i j}
$$

where $x_{i j}$ is the $\log _{2}$ expression level in sample $j, m_{i}$ is the overall mean $\log _{2}$ expression level, $G_{i j}$ is the indicator variable if sample $j$ is $\operatorname{Traf\sigma }^{-1}\left(G_{i j}=1\right.$ if sample $j$ is Traf $^{-1-}, 0$ otherwise), $T_{i j}$ is the indicator variable if sample $j$ is collected at day 10 , and $G T_{i j}$ is the genotype-time point interaction term $\left(G T_{i j}=1\right.$ if and only if $G_{i j}=1$ and $\left.T_{i j}=1\right), a_{i}, b_{i}$ and $r_{i}$ are the coefficients of the genotype and time point effects to be estimated, and $e_{i j}$ is the normally distributed error term. We determined whether probesets are differentially expressed between genotypes at day 6 (day 10) using the classifyTestsF function (nested F-test) from limma with default $P$-value cutoff 0.01 and generated Venn diagrams. Western blot analysis. Cell lysate preparation, SDS-PAGE, electrophoretic transfer, immunoblotting and development using enhanced chemiluminescence were accomplished as previously described ${ }^{41}$. To isolate ex vivo $\mathrm{T}_{\mathrm{E}}$ cells, OT-I cells were adoptively transferred into CD45.1 congenic mice followed by LmOVA infection. At day 7 post-infection donor cells were isolated $\left(\right.$ at $4{ }^{\circ} \mathrm{C}$ ) by MACS purification (Miltenyi Biotech). All antibodies for western analysis were purchased from Cell Signaling. P-AMPK measures phosphorylation at Thr 172.

31. Pearce, E. L. \& Shen, H. Generation of CD8 T cell memory is regulated by IL-12. J. Immunol. 179, 2074-2081 (2007).

32. Foulds, K. E. et al. Cutting edge: CD4 and CD8 T cells are intrinsically different in their proliferative responses. J. Immunol. 168, 1528-1532 (2002).

33. Elstrom, R. L. et al. Akt stimulates aerobic glycolysis in cancer cells. Cancer Res. 64, 3892-3899 (2004).

34. Deberardinis, R. J., Lum, J. J. \& Thompson, C. B. Phosphatidylinositol 3-kinasedependent modulation of carnitine palmitoyltransferase $1 A$ expression regulates lipid metabolism during hematopoietic cell growth. J. Biol. Chem. 281, 37372-37380 (2006).

35. Buzzai, M. et al. Systemic treatment with the antidiabetic drug metformin selectively impairs p53-deficient tumor cell growth. Cancer Res. 67, 6745-6752 (2007).

36. Karst, A. M., Dai, D. L., Cheng, J. Q. \& Li, G. Role of p53 up-regulated modulator of apoptosis and phosphorylated Akt in melanoma cell growth, apoptosis, and patient survival. Cancer Res. 66, 9221-9226 (2006).

37. Albert, M. H., Yu, X. Z., Martin, P. J. \& Anasetti, C. Prevention of lethal acute GVHD with an agonistic CD28 antibody and rapamycin. Blood 105, 1355-1361 (2005).

38. Wu, Z., Irizarry, R. A., Gentleman, R., Martinez-Murillo, F. \& Spencer, F. A modelbased background adjustment for oligonucleotide expression arrays. J. Am. Stat. Ass. 99, 909-917 (2004).

39. Gentleman, R. C. et al. Bioconductor: open software development for computational biology and bioinformatics. Genome Biol. 5, R80 (2004).

40. Smyth, G. K. in Bioinformatics and Computational Biology Solutions Using R and Bioconductor (eds Gentleman, R., Carey, V., Dudoit, S., Irizarry, R. \& Huber, W.) 397-420 (Springer, 2005).

41. Kobayashi, T. et al. TRAF6 is a critical factor for dendritic cell maturation and development. Immunity 19, 353-363 (2003). 